\section{The Forbidden Fruit}

Dear Reader,

The German car industry, often much criticized as being oh-so-backward, is getting into position by trying its luck with partnerships. A recent example: Bosch is hooking up with Microsoft and wants to use their Azure Cloud environment to create a software platform for the "seamless networking of vehicles." The goal of the cooperation is to continuously develop vehicle software and to update the vehicle computers with it. This will completely remove the situation where post-SOP vehicles are in a more or less frozen state of capabilities and functions. They will then be, to coin a phrase, "panta rhei" until the end of their lifecycle. The eternal flow of new software sets, adjustments and capabilities ranging from driver assistance up to safety-focused functions such as braking parameters or damping characteristics will mean an almost complete shift away from the definition of homologation.

In parallel to Microsoft, Amazon Web Services are also very popular with OEMs; so too, on other levels, are service providers such as T-Systems or NTT. The areas of application of these data-mining partnerships range from development to manufacturing, or the implementation of automated driving; and naturally topics such as OTA and cybersecurity center are also relevant. Against this backdrop, a profound metamorphosis of the vehicle and also the relationship between automotive manufacturer and supplier can be noted. In parallel to the electrification of the vehicles, the fourth industrial revolution is occurring in vehicle technology: "The future is electric" is an oft-cited phrase, but at the same time, the future is above all electronic and data-driven and not just in the context of vehicle technology. This development will not just require a suitable backbone or middleware in the vehicle, but also an ecosystem to handle and exploit the massive amounts of data. Drawing parallels to computer-generated Bitcoins and their enormous energy requirements can fill one with trepidation regarding the consumption of resources in the brave new world of highly automated, electrically powered vehicles. WLTP on its own may no longer be a standard for fuel consumption over $100 \mathrm{~km}$ : The trend toward clean electric vehicles, currently regarded as a panacea and extended with HAD systems, can also easily turn out to be a bite into the forbidden fruit when it comes to energy demand. And all this even before Apple launches its anticipated iCar on the market.

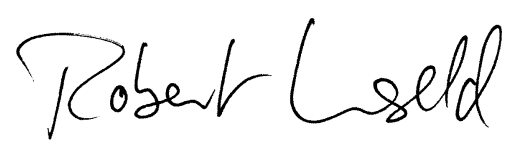

Robert Unseld Responsible Editor

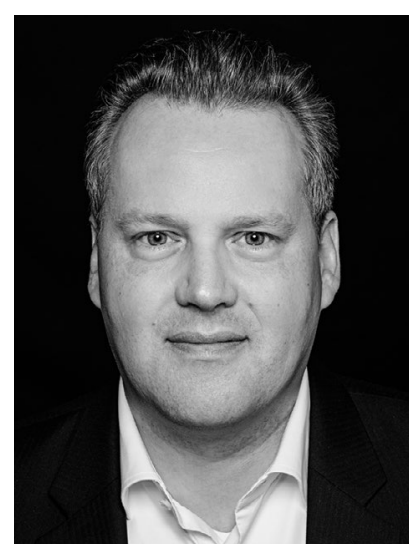

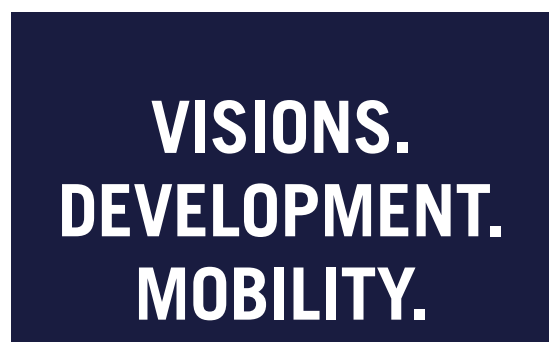

The world of mobility is changing. The challenge facing automotive developers is to acquire the knowledge they will need tomorrow without losing sight of future technologies. The complexity of the mobility transformation process calls for groundbreaking solutions. ATZelectronics provides the latest information from across the entire spectrum of automotive electronics. Take advantage of the interactive e-magazine and benefit from the extensive information in our unique online archive, which gives you the option of downloading PDF files.

\section{$A T Z$ electronics}

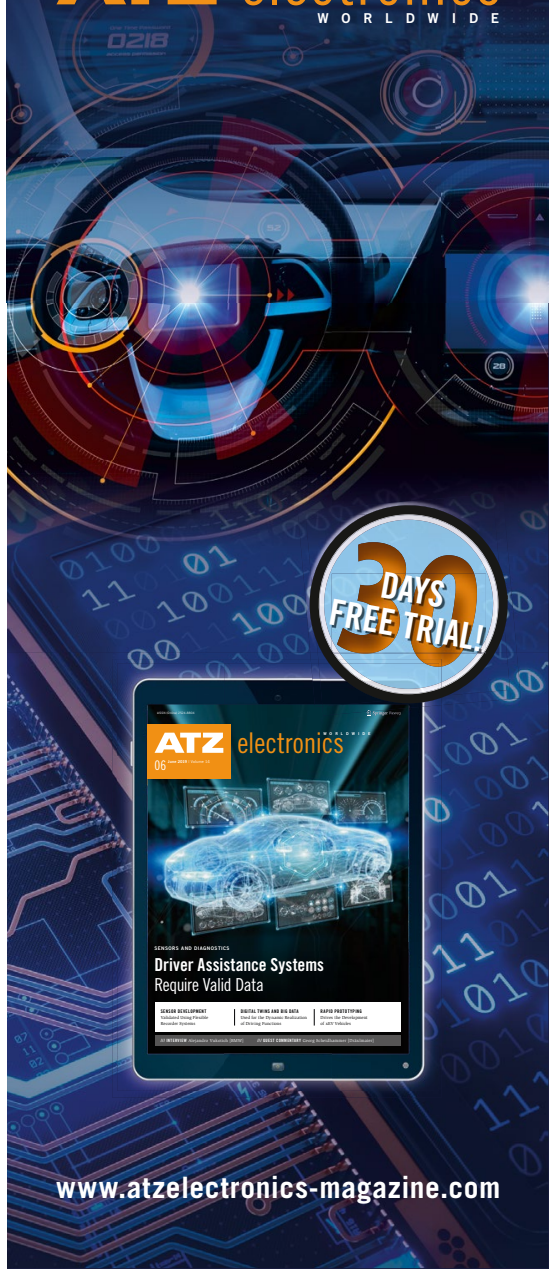

